\title{
Relationships between and sensitivities of the galvanic skin reflex and two indices of peripheral vasoconstriction in man
}

\author{
J. J. FUREDY AND YVETTE GAGNON
}

From the University of Toronto, Canada, and Indiana University, U.S.A.

Contrary to some views on the neurological relationship between the galvanic skin and peripheral vasoconstrictor reflexes (for example, Carmichael, Honeyman, Kolb, and Stewart 1941; Goadby and Goadby, 1936, 1949), Prout (1967) has suggested that the post-ganglionic pathways for the two responses are mutually independent. In support of this neurological independence, Prout has claimed behavioural independence for the two responses-that they 'may fluctuate independently in size and one reflex may occur in the absence of the other' (Prout, 1967, p. 323).

Prout's preparation, continuous simultaneous recordings of the two responses, is ideal for obtaining the data relevant to his claim. However, the presentation of the data itself is less than optimal, since only selected examples are provided without either any quantification or any specification of the criteria used for abstracting the relevant features from the continuous, surface-physiological recordings. When data are intended to settle a controversial issue like the present one of response independence, it is especially important to note that the technique of selecting illustrative instances to describe the data may produce examples which are representative more of the investigator's initial preconceptions than of the data as a whole. This technique, then, although valuable for generating hypotheses, needs to be supplemented by more systematic descriptive methods for testing those hypotheses. One aim of the present study, therefore, was to provide systematic and quantified evidence regarding the behavioural independence question by first measuring the galvanic-skin and vasoconstrictor response magnitudes to various stimuli, and then using the descriptive-statistical tool of correlation to assess the degree to which the two reflexes were independent.

The second aim of this study was to assess the relationship between two commonly used indices of the peripheral vasoconstrictor reflex, indices which are based, respectively, on measuring blood volume
(BV) and pulse volume (PV). The BV-based measure uses a DC-coupled arrangement, and vasoconstriction is represented by a shift in the baseline of the record in a given direction; the PV-based measure employs an AC-coupled arrangement, and vasoconstriction is represented by a decrease in the amplitudes of successive systolic pulses. Presumably for reasons of convenience, investigators have typically measured vasoconstriction either only in BV terms (for example, Zimny and Miller, 1966), or only in PV terms (for example, Prout, 1967; Furedy, 1968). One requirement for the validity of this convenient practice of using $\mathrm{BV}$ and $\mathrm{PV}$ as alternative measures of vasoconstriction is that the two indices be correlated hence the interest in this study in the relationship between the two indices.

Another requirement for the alternative use of the $\mathrm{BV}$ and $\mathrm{PV}$ indices is that the two response measures be equally sensitive in detecting such stimulus contrasts as the presence versus absence of a stimulus, or a strong (for example, shock) versus weak (soft tone) stimulus. To assess this question, the present study not only measured both indices from the same point on the same subject (Uno and Grings, 1965), but it also incorporated the additional desideratum of providing separate recording channels with DCand AC-coupling for the two indices. Such an arrangement allows optimal and independent settings for each measure, thus avoiding the difficulties involved in using the same tracing for both measures (see, for example, Brown, 1967, p. 57). The relative BV and PV sensitivities to stimulation were assessed by a primitive application of signal-detection methods (for example, Tanner and Swets, 1954) which are concerned with assessing the degree to which signal + noise is detected or discriminated from noise alone. For the present application, letting $\mathrm{X}$ stand for signal + noise and $\mathrm{Y}$ stand for noise alone, the tests allowed determination of how well a given index discriminated between the following $\mathrm{X}$ vs. $\mathrm{Y}$ stimulus contrasts: (a) WSv $\overline{\mathrm{W}, \mathrm{S}}$ the presence vs. absence of a weak stimulus; (b) 
SSv $\overline{S S}$, the presence vs. absence of a strong stimulus; (c) SSvWS, strong vs. weak stimulus; (d) CS+vCS-, a conditioned stimulus (CS) paired with an unconditioned stimulus (UCS) in classical (Pavlovian) conditioning vs. a CS which had not been so paired. In addition, the same sensitivity tests were performed for the galvanic-skin response (GSR). The purpose was to obtain systematic evidence concerning a view which has hitherto been based on what are perhaps subjectively convincing, but nevertheless informal, laboratory observations: that the GSR is a better signal detector than the vasoconstrictor reflex.

\section{METHOD}

SUBJECTS The subjects $(S s)$ were 62 Indiana University undergraduates who received introductory psychology course credit as well as the possibility of earning up to $\$ 1.50$.

APPARATUS The $S$ was seated in a padded armchair placed in an electrically shielded room separate from the experimenter $(E)$ and the main apparatus. There was intercom contact between $E$ and $S$, while a peep-hole also allowed $E$ to observe, without $S$ 's knowledge, whether $S$ 's eyes were open. Brush-crystal earphones on $S$ delivered a $60 \mathrm{db}$ spl white masking noise which was continuous except when a $300 \mathrm{msec}, 2,000 \mathrm{~Hz}, 80 \mathrm{db}$ tone was delivered. For the $300 \mathrm{msec}$ light stimulus, the illumination from a ceiling lamp was increased from 1.5 to 3.3 foot lamberts as measured with a Macbeth Illuminometer directed at the wall in front of $S$ at eye level. The shock source was AC and constant-current, with a maximum of $3 \mathrm{~mA}$, designed and constructed at Indiana University. The $200 \mathrm{msec}$ shock was increased in intensity from 1 to $2 \mathrm{~mA}$ in 1/3-mA steps over the first three shock trials, and was delivered through a Turskytype concentric electrode (Tursky, Watson, and O'Connell, 1965) affixed to the ventral surface of the right forearm. All temporal intervals were controlled by electronic timers, while the nature of the trials and the inter-trials intervals were programmed through a Western Union tape transmitter.

A photoelectric transducer attached to the tip of the left index finger was used to obtain the vasoconstrictor BV and PV indices. The BV pickup was direct coupled (DC) with its own separate tracing and pen. On the infrequent occasions when the record was close to going off scale, the pen was rebalanced during a period between stimulus presentations when no response measurement was to be carried out. The PV tracing was obtained from the same transducer, but was AC coupled with its own separate tracing and pen. This arrangement permitted optimal sensitivity control for each measure. An Offner Type-R dynograph run continuously at a paper speed of $5 \mathrm{~mm} / \mathrm{sec}$ was used to amplify and record the signal from the transducer.

The GSR was picked up by a Fels Dermohmmeter, with a current of $70 \mathrm{~mA}$ D.C. imposed across zincmanganese-dioxide electrodes attached to $S$ 's left palm and upper arm. Contact with the palmar surface was made through a $5 \%$ saline-soaked cotton ball of approximately 1 sq. in., while the reference electrode was placed over a saline-moistened rubber sponge of approximately $2 \times 6 \mathrm{sq}$. in., which was itself attached to the outer lateral surface of the left arm about 4 in. above the elbow.

PROCEDURE The present study was part of a classical differential conditioning experiment with tone and light as CSs, shock as UCS, and GSR and vasoconstrictor PV as dependent autonomic variables. The data reported here are based on a subset of $S$ s which consisted of all those $S \mathrm{~s}$ for whom vasoconstrictor BV was measured in addition to PV and GSR. The interval between CS and UCS onsets was $750 \mathrm{msec}$, but for 31 'forward' $S \mathrm{~s}$ the CS preceded the UCS, while for the remaining 'backward' $S$ s the UCS preceded the CS. Three consecutive 10-trial stages each consisted of: (a) four pairings of the reinforced CS (CS +, tone or light) with the UCS, these pairings constituting the strong stimulus (SS) for this report; (b) one CS + trial without UCS; (c) five non-reinforced CS (CS -, light or tone) presentations which, for this report, are instances of the weak stimulus (WS). Within stages, the order of the three types of trials was unsystematic, and the intervals between all trials varied randomly between 30,40 , and $50 \mathrm{sec}$.

\section{RESULTS}

RESPONSE MEASURES The vasoconstrictive change i digital pulse volume (PV) is a decrease in pulse magnitude. The response definition, based op successively measured systolic pulse magnitudes, has been described and used elsewhere (Furedy, 1968) The definition seeks to control for the brief and nonstimulus connected arhythmic pulse-magnitude changes which are characteristic of PV recordings, by requiring that response initiation and termination occur for longer than two-pulse periods. Specifically, response initiation was looked for from 2 to $8 \mathrm{sec}$ after stimulus onset, and defined as occurring at the first (base) pulse, I, for which either (a) I was followed by pulses $\mathbf{J}$ and $\mathrm{K}$ where $\mathrm{I}>\mathrm{J}>\mathrm{K}$, or (b) I was followed by pulses, J, K, and $\mathrm{L}$ where I $>\mathbf{J}>\mathbf{L}$. Response termination was taken to have occurred at pulse $\mathrm{T}$, which followed $\mathrm{I}$ and $\mathrm{J}$, and which itself was followed by pulses $U$ and $V$ such that $\mathrm{T} \leqslant \mathrm{U} \leqslant \mathrm{V}$. The amount of change was the algebraic difference between $\mathrm{I}$ and $\mathrm{T}$ expressed as a percentage of $I$, so that the PV response was a \% volume-pulse change measure.

To ensure comparability, the BV response was defined in terms of the same criteria as the PV response, the only difference being that the midpoints of the systolic pulses, rather than their magnitudes, served as the basic data, which in turn implied that vasoconstriction was measured in terms of a shift in midpoint position rather than a change in pulse 
magnitude. The amount of BV change, as represented by the difference between the response-onset and response-termination midpoints, was converted into millivolts according to the sensitivity settings used for each $S$ on each trial.

The GSR, a drop in skin resistance initiated between 1 and $5 \mathrm{sec}$ after stimulus onset, was transformed into conductance change $(\triangle C)$ in micromhos. For all three response definitions, the stimulus onset on paired presentations of tone or light and shock was taken to be the onset of the first stimulus.

SENSITIVITY MEASURE Response sensitivity was assessed by determining percentage detection ( $\% \mathrm{D})$ indices which reflected the response $(R)$ to the $X$ and $\mathrm{Y}$ components of the various $\mathrm{X} v$ s. $\mathrm{Y}$ stimulus contrasts. Depending on the relative size of $\mathbf{R}$ to $\mathbf{X}$, $\mathbf{R}_{\mathbf{x}}$, on a given trial, $\% \mathrm{D}$ was defined by alternative equations: for $R_{X}>R_{Y}, \% D=100\left(R_{X}-R_{Y}\right)$ / $R_{X}$, while for $R_{X}<R_{Y}, \% D=100\left(R_{X}-R_{Y}\right) / R_{Y}$. This index is symmetrically weighted for $R_{x}$ and $R_{Y}$ scores, varies about zero from +100 to -100 , and the average signal-detectability of a given form of the response is indicated by the size of any positive mean $\% \mathrm{D}$ score.

COMPARISONS OF RELATIVE SENSITIVITIES Figure 1 shows the sensitivities of the measures over the $X$ vs. $Y$ contrasts. In Panels $A$ and $B$, where $Y$ was the

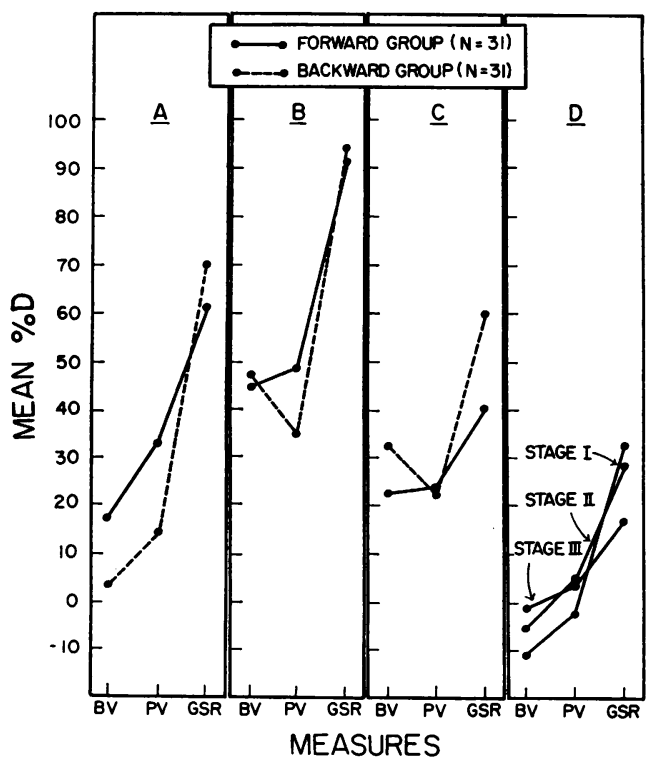

FIG. 1. Mean $\% D$ values of the three measures ( $B V$, $P V$, and GSR) on the four $X$ vs. $Y$ contrasts: $W S v \overline{W S}$ (Panel A); SSv $\overline{S S}$ (Panel B); SSvWS (Panel C); $C S+\mathrm{v} C S-$ for the forward group only (Panel D). absence of the stimulus, control periods commencing $12 \mathrm{sec}$ before stimulus onset were used to measure the 'response' $\left(R_{Y}\right)$, the definition of which was identical with that of the response following the stimulus $(\mathbf{R x})$. The $\% \mathrm{D}$ scores in Panels $\mathrm{A}, \mathrm{B}$, and $\mathrm{C}$ are based on the first three WS and SS trials in the trial series. The CS + vCS - contrast in Panel D was restricted to the forward group, because only under this CS-UCS condition was there evidence of highly reliable differential conditioning (response to $\mathrm{CS}+$ greater than that to $\mathrm{CS}-$ ) in the GSR and PV data based on all $80 \mathrm{Ss}$ of the main experiment. Response measures to CS + ( $\left.\mathbf{R}_{\mathbf{x}}\right)$ for Stages I, II, and III in Panel D are based on the three unpaired $\mathrm{CS}+$ trials, while the CS - responses $\left(\mathrm{R}_{\mathrm{Y}}\right)$ were obtained from CS - trials proximal to the CS + trials in each stage. Double-classification analyses of variance (McNemar, 1955), indicated significant effects due to measures on all four contrasts: $F(2,120)=28.789,33.665$, and 10.703 for the first three panels, $P<0.001 ; F(2,60)=10.256$, $P<0.01$ for Panel D. No other main effects (groups or stages) or any interactions approached significance for any of the contrasts, $P>0 \cdot 2$. Separate analyses of the measures effects indicated that for all contrasts the mean GSR $\% \mathrm{D}$ significantly exceeded the higher of the two plethysmographic $\%$ D values: $F(1,60)=42.992$ and 100.071 for Panels A and B, respectively, $P<0.001 ; F(1,60)=$ 10.256, $P<0.01$ for Panel $C ; F(1,30)=6.260$, $P<0.05$, for Panel D. However, the differences between BV and PV \%D values did not approach significance in any of the contrasts, $P>0 \cdot 2$.

INTERCORRELATIONS OF MEASURES Based on the $\% D$ scores obtained from the above four $\mathrm{X}$ vs. $\mathrm{Y}$ contrasts, product-moment correlations (McNemar, 1955) were computed between pairs of the three autonomic measures. Table I presents these correlations and the significance levels associated with each. It will be noted that the BV-PV correlations (relationship between vasoconstrictor-reflex indices) are all positive, and all except one are significant at

TABLE I

PRODUCT-MOMENT CORRELATIONS BETWEEN PAIRS OF MEASURES BASED ON $\%$ D VALUES FOR THE VARIOUS $X$ VS. $Y$ STIMULUS CONTRASTS

\begin{tabular}{|c|c|c|c|}
\hline & \multicolumn{3}{|c|}{ Pairs of measures } \\
\hline $\begin{array}{ll}\text { WS } v \overline{\text { WS }} & \\
\text { SS } v \text { SS } \\
\text { SS } v \text { WS } \\
\text { CS }+v \text { CS }- \text { Stage I } \\
& \text { Stage II } \\
& \text { Stage III }\end{array}$ & $\begin{array}{l}B V-P V \\
0.345 \dagger \\
0.356 \dagger \\
0.549 \ddagger \\
0.361^{*} \\
0.559 \ddagger \\
0.336\end{array}$ & $\begin{array}{c}B V-G S R \\
-0.090 \\
0.048 \\
0.135 \\
0.154 \\
-0.135 \\
-0.136\end{array}$ & $\begin{array}{r}P V-G S R \\
0.227 \\
-0.136 \\
0.168 \\
-0.205 \\
0.051 \\
0.071\end{array}$ \\
\hline
\end{tabular}

Two-tailed *P<0.05, $+P<0.01$, and $\ddagger P<0.001$. 
beyond the $5 \%$ level of confidence. In contrast, the BV-GSR and PV-GSR correlations (relationship between the vasoconstrictor and galvanic-skin reflex) are all smaller than any of the BV-PV correlations, as well as being negative almost as often as they are positive. The relationship between the vasoconstrictor and galvanic-skin reflexes, therefore, seems to be one of independence.

\section{DISCUSSION}

The lack of any significant correlations between the GSR and either the BV- or PV-based index of the vasoconstrictor reflex provides systematic evidence to support Prout's (1967) more informally based claim that the two reflexes are behaviourally independent. In turn, the behavioural independence of these surface-physiological measures supports Prout's position of neurological independence with respect to the post-ganglionic pathways for the human galvanic-skin and peripheral-vasoconstrictor reflex.

On the other hand, the correlations between the BV- and PV-based indices of vasoconstriction suggest that these two indices are related. That the obtained correlations, though significant in all but one case, were not high is probably accounted for by the low reliability of both vasoconstrictive measures, since the reliabilities of any two measures set an upper bound to the degree of obtained correlation between them (McNemar, 1955). Hence, the fact that all except one of the obtained correlations were significantly positive gave some support to the convenient practice of using BV and $\mathrm{PV}$ as alternative measures of human digital vasoconstriction. Further, and perhaps stronger, support for this practice was provided by the present evidence that the two measures seemed to be equally sensitive in detecting a number of different stimulus contrasts. This BV-PV substitutability is important not only for the convenient practice of using the indices as alternative measures, but also for utilizing analogue-to-digital conversion methods for measuring vasoconstriction. Such methods, which eliminate both human drudgery and bias, are more readily applicable to the DC-coupled BV index, provided that the pulses can be damped out from the record. In that case, the sampling rate required for digitization of the positional shifts of this analogue is much lower than that needed for registering the pulse magnitudes obtained from the AC-coupled PV analogue.

Finally, it bears emphasis that while there was no difference in BV and PV sensitivities on any of the stimulus contrasts, the GSR was significantly more sensitive than either vasoconstrictor measure on all four contrasts. These results constitute systematic support for a view that is probably widely accepted on the basis of informal laboratory observations: that, in humans, the GSR is markedly superior to the peripheral vasoconstrictor reflex as a surfacephysiological detector of signal over noise.

\section{SUMMARY}

Two indices of the vasoconstrictor reflex based, respectively, on blood-volume (BV) and pulsevolume (PV) change, as well as the galvanic skin response, have been simultaneously recorded from 62 healthy undergraduates exposed to brief tone, light, and mild-shock stimulations.

Computations of several correlations between the galvanic-skin and vasoconstrictor reflexes yielded near-zero and non-significant values, indicating that, in man, the two types of reflexes are behaviourally, and probably neurologically, independent.

In contrast, the positive and significant correlations obtained between the two indices of the vasoconstrictive reflex support the practice of using the BV- and PV-based measures as alternative indices of vasoconstriction. Further support for this practice is provided by the finding that the two indices were equally effective as signal-to-nois detectors on all examined contrasts. In view of the 응 support for BV-PV substitutability as indices of vasoconstriction, the advantages of the BV-baseg $c$ measure for analogue-to-digital conversion an discussed.

Finally, comparisons on all stimulus contras indicate that, as a signal-to-noise detector, the galvanic-skin reflex is markedly superior to the peripheral vasoconstrictor reflex.

This investigation was supported by Contract No. ڤँ 908 (15), Office of Naval Research (Roger W. Russell, $\frac{\Phi}{\varnothing}$ Project Director). The authors are indebted to Dr. David C. Raskin for originally suggesting the idea of recording BV and PV through separately coupled tracings; 3 Dr. Daniel E. Berlyne, Dr. John A. Stern, and Dr. John C. Ogilvie for suggestions regarding the preparation of this? report; Mrs. Nancy L. Garrett for assistance in data reduction and analysis; John K. Stevens for technical advice and assistance. Additional work for the preparation of this report was supported by Grant APA 303 118-80 to the senior author from the National Research Council of Canada.

\section{REFERENCES}

Brown, C. C. (1967). Methods in Psychophysiology. Williams and $D$ Wilkins: Baltimore.

Carmichael, E. A., Honeyman, W. M., Kolb, L. C., and Stewart, W. R. (1941). A physiological study of the skin resistance $N$ response in man. J. Physiol. (Lond.), 60, 316-321.

Furedy, J. J. (1968). The human orienting reaction as a function of $\mathrm{N}$ electrodermal versus plethysmographic response modes and single versus alternating stimulus series. J. exp. Psychol., 77, $\mathrm{W}$ $70-78$. 
Goadby, K. W., and Goadby, H. K. (1936). Simultaneous photographic records of the potential and resistance effects of the psycho-emotive response. J. Physiol. (Lond.), 86, 11-13P.

(1949). The nervous pathway of the psycho-galvanic skin reflex. Ibid., 109, 177-184.

McNemar, Q. (1955). Psychological Statistics. 2nd ed. Wiley: New York.

Prout, B. J. (1967). Independence of the galvanic skin reflex from the vasoconstrictor reflex in man. J. Neurol. Neurosurg. Psychiat., 30, 319-324.
Tanner, W. P., Jr., and Swets, J. A. (1954). A decision-making theory of visual detection. Psychol. Rev., 61, 401-409.

Tursky, B., Watson, P. D., and O'Connell, D. N. (1965). A concentric shock electrode for pain stimulation. Ibid., 1, 296-298.

Uno, T., and Grings, W. W. (1965). Autonomic components of orienting behavior. Ibid., 1, 311-321.

Zimny, G. H., and Miller, F. L. (1966). Orienting and adaptive cardiovascular responses to heat and cold. Ibid., 3, 81-92. 